\title{
TAREAS 2.0 PARA ELAPRENDIZAJE Y EVALUACIÓN DE SEGUNDAS LENGUAS EN ENTORNOS VIRTUALES DE APRENDIZAJE.
}

\section{TASKS 2.0 FOR LEARNING AND EVALUATION OF SECOND LANGUAGES IN VIRTUAL ENVIRONMENTS.}

\author{
Dr. Esteban Vázquez Cano \\ evazquez@edu.uned.es
}
Universidad Nacional de Educación a Distancia. Facultad de Educación. Departamento de Didáctica, Organización Escolar y Didácticas Especiales. (UNED).
Juan del Rosal 14, 28040, Madrid (España)

Este artículo presenta una investigación contextualizada en el Máster universitario en Tecnologías de la Información y la Comunicación en la Enseñanza y Tratamiento de Lenguas de la UNED en el que se analiza la didáctica y la evaluación de segundas lenguas en entornos virtuales de aprendizaje. El objetivo fundamental es proporcionar claves esenciales ante el reto de desarrollar una didáctica y evaluación de las segundas lenguas con nuevas tareas y herramientas más dinámicas y colaborativas que deben llevar aparejado un cambio didáctico y metodológico que integre nuevas formas de desarrollar y evaluar las destrezas lingüísticas a través de tareas 2.0.

Palabras clave: Entornos virtuales de aprendizaje, web 2.0, enseñanza de segundas lenguas, aprendizaje colaborativo y ubicuo.

This paper presents a contextualized research in the Master degree ICT in Language Education and Treatment which is organized by the UNED, and analyses the teaching and assessment of second language learning in virtual environments. The main objective is to provide essential clues to the challenge of developing and evaluating second language teaching by means of new tasks and more dynamic and collaborative tools in order to develop new methodologies that integrate ways of developing and assessing the language skills through 2.0 tasks. Keywords: Virtual learning environments, web 2.0, second language teaching, collaborative and ubiquitous learning. 


\section{Introducción.}

Presentamos en este artículo una investigación sobre metodologías y procesos evaluadores fundamentados en tareas 2.0 en entornos virtuales de aprendizaje en el Máster universitario en Tecnologías de la Información y la Comunicación en la Enseñanza y Tratamiento de Lenguas de la UNED. El objetivo fundamental es analizar la utilidad de las herramientas digitales para el desarrollo de tareas 2.0 que suponen un desarrollo más efectivo de la metodología y evaluación de segundas lenguas en Entornos Virtuales de aprendizaje (EVA). La didáctica y evaluación de segundas lenguas son dos aspectos en constante revisión y, en los últimos años, se han estado desarrollando numerosas propuestas que avanzan en la comprensión de la habilidad lingüística de los aprendientes (Arnold, 2007; Campbell, 2007; Coniam, 2008; de Freitas \& Neumann, 2009; Harrison \& Thomas, 2009). La incorporación de las Tecnologías de la Información y la Comunicación (TIC) ha cambiado sustancialmente el paradigma de actuación metodológica en la enseñanza de segundas lenguas y, por extensión, las tareas y pruebas asociadas a la evaluación de competencias y habilidades lingüísticas en EVA. La aplicación del tratado de Bolonia y la creación del Espacio Europeo de Educación Superior (EEES) han supuesto una revolución en el modelo docente. La organización del proceso de enseñanza-aprendizaje bajo el nuevo enfoque ofrece al alumnado un papel protagonista de su propio aprendizaje y al profesor el papel de facilitador de que este proceso se produzca, reduciendo a la vez su papel central como transmisor del conocimiento (Valle-López, 2010). La tecnología de aprendizaje dominante se articula a través de plataformas tecnológicas de distribución del aprendizaje (Learning Management System, LMS). Este tipo de software lo encontramos en la inmensa mayoría de entornos virtuales de aprendizaje de nuestras universidades, en tres formatos: 1) versión plataformas privativas basadas en código propietario: herramientas como WebCT y Blackboard; 2) versión software libre: Moodle, Atutor o Bodington, entre otras; y 3) versión de entornos virtuales de aprendizaje desarrollados por la propia universidad como la plataforma aLF de la UNED. Este nuevo contexto tecnológico y colaborativo y su aplicación en la enseñanza y aprendizaje de segundas lenguas precisa de nuevas formas de afrontar la metodología de la enseñanza de lenguas desde una perspectiva más dinámica e interactiva y en donde los EVA suponen un reto para el docente en una doble faceta: metodológica y evaluadora. Si bien las dinámicas web 2.0 están propiciando nuevas formas de enseñar y aprender segundas lenguas; los procesos metodológicos y evaluadores en EVA precisan de una revisión teórico-práctica constante.

\subsection{Didáctica y evaluación 2.0 en segundas lenguas.}

La didáctica para el desarrollo del proceso de enseñanza-aprendizaje de las segundas lenguas en Europa se encuentra en un momento de cambio de perspectiva; propiciado por la publicación hace ya nueve años del Marco Común Europeo de Referencia para las lenguas (MCER), y por su posicionamiento definitivo a favor de una perspectiva orientada a la acción (Bugler \& Hunt, 2002; Copley, 2007; Boneu, 2007; Ellis, 2009; Willis \& Willis, 2009). 
Con el desarrollo de los entornos virtuales de aprendizaje posicionamos la docencia de segundas lenguas ante un entorno sociotecnológico formado por individuos usuarios de la lengua y de la tecnología webque interactúan entre sí para desarrollar destrezas y habilidades comunicativas a través de tareas comunicativas digitales tareas 2.0- (Area \& Adell, 2009; Ortega, 2009; Reeder, 2010; Solano \& Sánchez, 2010; Vázquez-Cano \& Sevillano, 2011). La base metodológica del aprendizaje de segundas lenguas por tareas se fundamenta en las teorías lingüísticas comunicativas o funcionales, pero también en los enfoques educativos constructivistas actuales. Es evidente que entre las nuevas funciones del profesorado se encuentra la de integrar e incorporar los medios a la enseñanza de forma efectiva y a favor de la optimización del aprendizaje. Por ello, su formación y perfeccionamiento orientados al uso y manejo de las TIC es una de las piedras angulares que va a condicionar dicha integración (de Benito \& Salinas, 2008; Cabero, 2010; del Moral \& Villalustre, 2012).

La didáctica basada en el desarrollo de tareas 2.0 se basa principalmente en los siguientes principios: aprender haciendo, aprender interactuando, aprender buscando y aprender compartiendo. Este tipo de aprendizaje se convierte en más significativo (Jonassen, 1995; Nevgi \& Tirri, 2003; Thomas \& Reinders, 2010). Al diseñar el contenido de las tareas de aprendizaje 2.0, el docente debe orientar su didáctica hacia una dimensión más grupal y, a la vez, colaborativa y debe fomentar una dimensión más interactiva del proceso de aprendizaje que favorezca un aprendizaje más informal. Los entornos virtuales de aprendizaje potencian el desarrollo de este aprendizaje informal a través de la participación y colaboración, así como con el aprendizaje ubicuo y móvil propiciado por los dispositivos digitales móviles (Sharples, 2006; Fallahkhair, Pemberton \& Griffiths, 2007; Lan, Sung \& Chang, 2007). Junto a estos principios, aparecen también los conceptos de colaboración y trabajo en red, ya que un educador/profesor en el siglo XXI debe, entre otros diversos medios y estrategias, dominar la capacidad de comunicar, crear y enseñar en red (Vázquez-Cano \& Sevillano, 2011).

Asimismo, Bachman (2000) enuncia los asuntos que considera que van a preocupar y a estudiarse en el campo de la evaluación de la lengua en el siglo XXI. Uno de los aspectos más determinantes de los asuntos propuestos es que se puede trabajar con algo más que los resultados de los exámenes, pudiendo ir más allá de la especulación acerca de su significado y de su uso, y en donde evaluación debe ser espejo de la metodología utilizada (Hislope, 2008). La evaluación de diferentes destrezas lingüísticas en EVA conlleva nuevos diseños metodológicos pero también nuevas formas de evaluar estas destrezas por medio de herramientas 2.0 que permitan observar la progresión y consecución de los diferentes indicadores y criterios de evaluación. Los principios en los que se debe sustentar la evaluación deben enfocarse hacia una evaluación formativa orientada a tareas 2.0 que desarrollen habilidades y destrezas lingüísticas necesarias en la adquisición de segundas lenguas.

\subsection{Objetivos de estudio.}

El contexto tecnológico en la enseñanza de segundas lenguas es un camino en constante cambio metodológico y evaluativo; para 
realizar una contribución proyectable en futuros contextos de enseñanza virtual, nos hemos planteado los siguientes objetivos:

- Conocer la apreciación del alumnado sobre qué herramientas 2.0 son más funcionales para la enseñanza de destrezas lingüísticas de segundas lenguas en EVA.

- Conocer la valoración del alumnado sobre la utilidad de las herramientas 2.0 en EVA para la evaluación de segundas lenguas.

\section{Metodología de la investigación.}

El presente trabajo se ha abordado como un estudio de caso desde la perspectiva de estrategia de intervención en el aula y posteriormente como herramienta para el desarrollo de la investigación, encuadrándose en una metodología doble de corte cualitativo y cuantitativo. El enfoque cualitativo ha ido encaminado a obtener información relevante para el análisis de las principales potencialidades de los EVA en la enseñanza de lenguas. De Pablos, Colas y Villaciervos (2010) mantienen que el uso o integración de las TIC en la educación es complejo y que, por este motivo, las investigaciones sobre esta temática se han ido reorientando hacia estudios de carácter cualitativo, que posibilitan un conocimiento más profundo sobre el impacto que producen. De modo que hemos analizado en profundidad una unidad microcontextual que busca, principalmente, la descripción y comprensión de un grupo reducido de participantes (Bolívar, Domingo \& Fernández, 2001) y sus apreciaciones sobre el uso de herramientas 2.0 en EVA para la enseñanza de segundas lenguas a través de sus aportaciones en foros de discusión en línea. Asimismo, hemos afrontado las expectativas de los estudiantes con respecto a qué recursos y herramientas 2.0 son más productivas en la didáctica y evaluación de segundas lenguas en EVA por medio de un estudio cuantitativo mediante un cuestionario para el que se ha aplicado el análisis factorial.

\subsection{Participantes y contexto.}

La muestra de estudiantes ha sido de 37 mujeres y 18 hombres lo que hace un total de 55 alumnos/as; con el siguiente perfil de edad y de estudios:

Como se puede apreciar en la Figura 1 y 2, la franja de edad mayoritaria oscila entre dos rangos prioritarios los 25 y 39 años con estudios universitarios de Licenciatura, principalmente, en alguna Filología. El contexto en el que hemos centrado el estudio de casos está contextualizado en el mismo entorno que se quiere analizar: los EVA en la asignatura de «Elaboración y Edición de materiales audiovisuales $»$ del «Máster universitario en Tecnologías de la Información y la Comunicación en la Enseñanza y Tratamiento de Lenguas >. El $87 \%$ del alumnado que realiza estos estudios es docente de primeras o segundas lenguas en ejercicio de diferentes etapas educativas; por lo que es altamente crítico con las metodologías y procesos evaluadores de segundas lenguas. Lo que nos posiciona ante una muestra de estudiantes con altas capacidades académicas y experiencia profesional en la enseñanza de segundas lenguas; lo que confiere a sus aportaciones un alto poder interpretativo.

\subsection{Recogida de información y análisis de datos.}

La recogida de datos cuantitativos se ha realizado a través de un cuestionario cuyo 


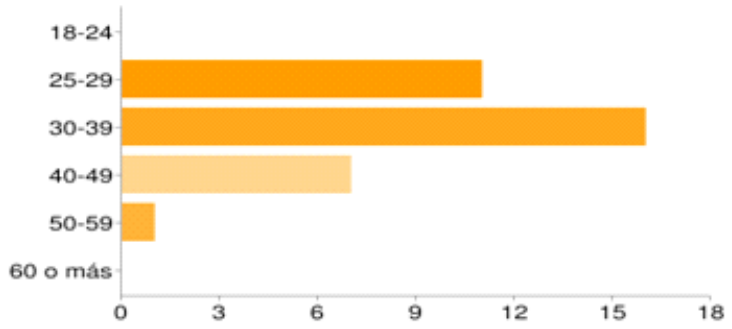

Figura 1. Rango de edad de los estudiantes de la muestra.

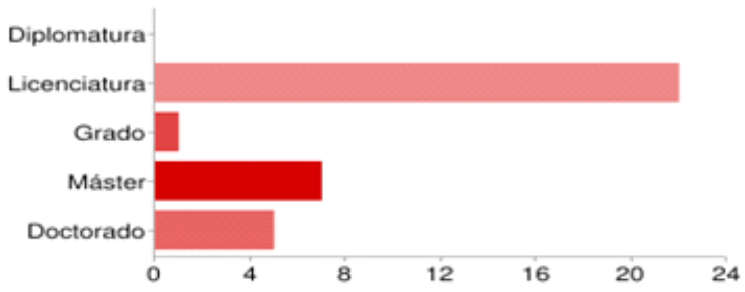

Figura 2. Estudios de los estudiantes participantes.

grupo de trabajo ha dado lugar a un cuestionario registrado como patente para el desarrollo de tareas 2.0 y de prácticas evaluadoras con el nombre: «Análisis de Entornos Virtuales de Aprendizaje para la Enseñanza de Lenguas»> (Puede realizarse su consulta en: http://breu.bulma.net/?114121). Asimismo, la recogida de datos cualitativos se ha realizado mediante el análisis de narraciones y ensayos críticos por medio de la herramienta virtual de foros. Esta actividad forma parte de la evaluación continua de la asignatura del Máster y se encaminó a recoger las apreciaciones del alumnado con respecto al uso de herramientas 2.0 en la enseñanzaaprendizaje de segundas lenguas y los procesos asociados a su evaluación; lo que muestra un gran valor para los propios actores (Chase, 1995). La participación en foros de discusión en línea como tarea académica dentro del desarrollo de la asignatura del Máster; así como la respuesta al cuestionario produce una narración extensa de tipo descriptivo y vivencial, en la que afloran los hechos y emociones que han sido más representativos y cruciales para los implicados. Promover la comprensión de los actores principales del proceso formativo es muy importante y supone un medio para mejorar la práctica educativa (Schön, 1992; Hernández \& Vergara, 2004). Los estudiantes han gozado de libertad para realizar sus narraciones, de forma abierta y crítica, desde un proceso dinámico y continuado, al tiempo, que compartían con el equipo docente, a lo largo del cuatrimestre, sus preocupaciones, inquietudes y logros a través de foros de discusión en la plataforma aLF de la UNED; 


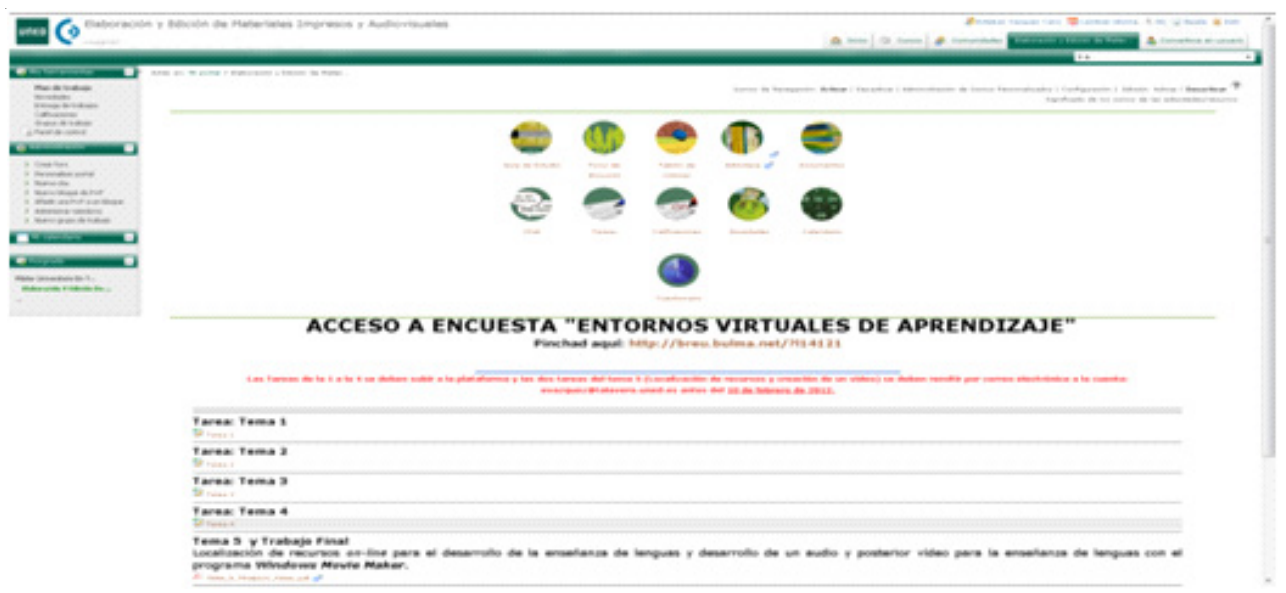

Figura 3. Página de inicio de la asignatura del Máster en aLF (UNED).

el entorno virtual de aprendizaje que desarrolla esta universidad para dinamizar los procesos de enseñanza-aprendizaje de sus estudios (ver Figura 3).

En cuanto a la validez del instrumento utilizado (foros de discusión), está condicionada por la propia veracidad de la información obtenida (Martínez-Miguélez, 2006). Por ello, como adelantamos, inicialmente se le ha facilitado al alumnado unas pautas básicas para estructurar el contenido de los ensayos críticos y los foros de discusión. Desde un principio y, a lo largo del proceso, se les fue incentivando para que desarrollasen con autenticidad las narrativas. Se establecieron dos foros de discusión en línea contextualizados en la didáctica y evaluación de segundas lenguas en EVA durante un periodo de tres meses. De hecho, Arandia, Alonso-Olea y Martínez-Domínguez (2010), encuentran que los procesos metodológicos de tipo dialógico contribuyen a que el alumnado tome conciencia de su aprendizaje y propicie la formación de un pensamiento argumentativo y crítico. Para elaborar los análisis de contenido de la información producida por los estudiantes en los foros, se utilizó el programa de "Analysis of Qualitative Data" (AQUAD), versión 5. La selección de la información introducida en el programa AQUAD se ha realizado, desde los datos brutos, atendiendo a su respectiva categorización primaria. El procedimiento informático ha requerido, como paso previo, su conversión al formato .txt, con archivos independientes para cada participante, con el objeto de poder desarrollar su codificación. Finalmente, se ha utilizado el programa EXCEL para concretar el recuento de frecuencias de las subcategorías, de forma individual (para cada participante y código de análisis) y globalmente (atendiendo a las respectivas subcategorías y al conjunto de los participantes). La codificación de los análisis permitió profundizar en el contenido de las narrativas, considerando tanto sus aspectos objetivables como los de carácter subjetivo y emocional (Clandinin \& Connelly, 1994). Los datos globales y desglosados se representan 


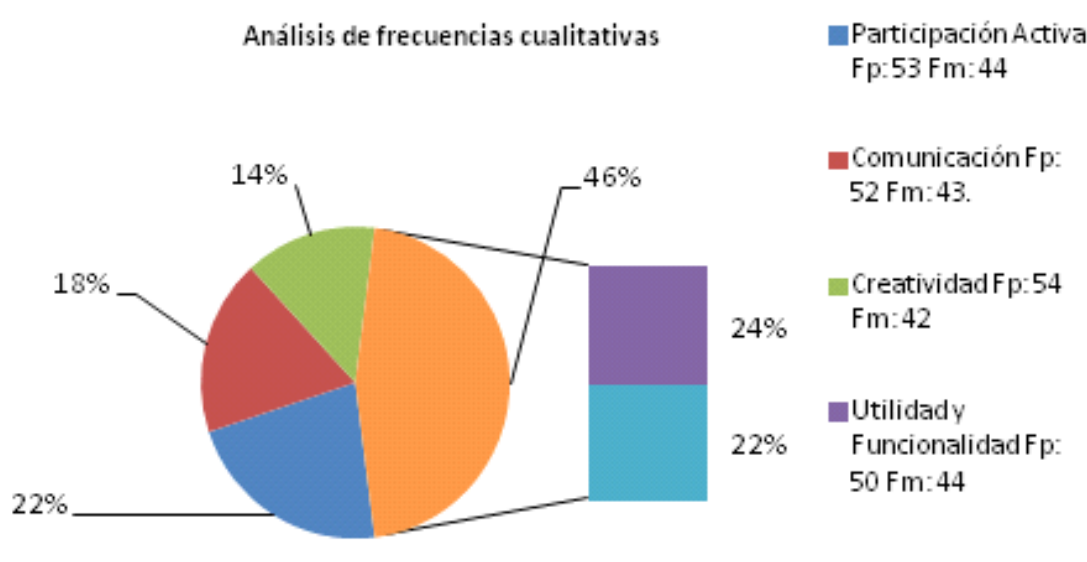

Figura 4. Análisis de frecuencias: principales potencialidades de los EVA en la didáctica de segundas lenguas.

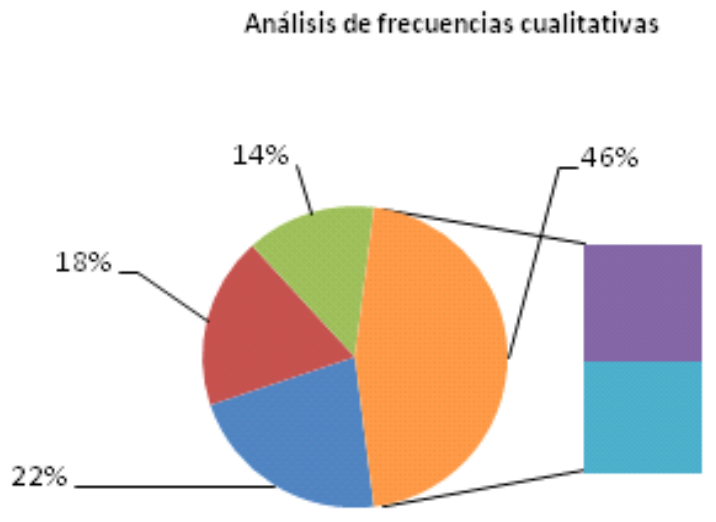

Coevaluación Fp:52 $\mathrm{Fm}: 41$

Autoevaluación Fp: $51 \mathrm{Fm}: 47$.

Evaluación continua Fp:54 Fm:42

$24 \%$

Evaluación por competencias Fp: 55 Fm: 49

$22 \%$
Evaluación sumativa

Fp:52 Fm: 44

Figura 5. Análisis de frecuencias: principales potencialidades de los EVA en la evaluación de segundas lenguas.

Píxel-Bit. Revista de Medios y Educación. $N^{o}$ 44. Enero 2014. ISSN: 1133-8482.

E-ISSN: 2171-7966. doi: http://dx.doi.org/10.12795/pixelbit.2014.i44.13 
a través de diferentes figuras en el apartado de resultados.

\section{Resultados.}

Las subcategorías se exponen de forma pormenorizada señalando el número de frecuencias de los participantes (fp) y el de la cantidad de manifestaciones (fm) que han emitido. $\mathrm{Su}$ indicador, permite conocer la preponderancia o particularidad que se registró con las respectivas subcategorizaciones. En cuanto a la fiabilidad de los resultados obtenidos, la clave para alcanzarla está asociada a la sistematización lograda en el desarrollo del proceso de investigación y a la consistencia alcanzada en los mismos (Hernández, Fernández \& Baptista, 2006). Los resultados más significativos de las unidades textuales analizadas en relación a las principales funcionalidades de las herramientas 2.0 en EVA para la didáctica y evaluación de segundas lenguas han sido categorizados en hiperónimos descriptivos y son los que se muestran en las dos figuras siguientes:

Asimismo, los resultados obtenidos de carácter cuantitativo mediante el cuestionario, se han analizado recurriendo a análisis descriptivos y tablas de contingencia que nos facilitaban frecuencias y porcentajes de las variables analizadas. De los estudios realizados, se obtienen los resultados que se muestran, a continuación, organizados en función de los objetivos de nuestra investigación. En primer lugar, abordaremos las expectativas de los estudiantes con respecto a la funcionalidad de las herramientas virtuales en el desarrollo de didácticas con base en tareas 2.0 en EVA; para posteriormente valorar la funcionalidad de las estrategias, recursos y herramientas 2.0 en la evaluación.

\subsection{Expectativas de los estudiantes sobre} la utilidad didáctica de herramientas 2.0 en EVA.

Hemos analizado cuáles eran las valoraciones que muestran los estudiantes del Máster con respecto a la funcionalidad y utilidad de las herramientas TIC en el desarrollo de procesos de enseñanzaaprendizaje de segundas lenguas basados en EVA. Se estableció en el cuestionario la posibilidad de elegir entre las herramientas y recursos web 2.0 que se muestran en la Tabla 1; los estudiantes reseñaron como herramientas más útiles en el desarrollo de metodologías 2.0 para la enseñanza de segundas lenguas en EVA, las siguientes (sólo se han reseñado frecuencias iguales o superiores al $40 \%$ de las intervenciones):

Los resultados se pueden agrupar en tres ámbitos. Un primer ámbito que está conformado por aquellas herramientas 2.0 que son más funcionales para el desarrollo de todas las destrezas. Un segundo ámbito constituido por las herramientas 2.0 que son más útiles para el desarrollo de la expresión oral y comprensión auditiva o de la expresión escrita y la comprensión lectora; y un último ámbito, compuesto por las herramientas 2.0 con poca funcionalidad en las cuatro destrezas.

\subsection{Expectativas de los estudiantes sobre la utilidad en la evaluación de herramientas 2.0 en EVA.}

Hemos analizado cuáles eran las valoraciones que muestran los estudiantes del Máster con respecto a la funcionalidad y 


\begin{tabular}{|c|c|c|c|c|}
\hline \multirow{2}{*}{$\begin{array}{c}\text { HERRAMIENTAS } \\
2.0\end{array}$} & \multicolumn{4}{|c|}{ DESTREZAS LINGÜÍSTICAS } \\
\hline & Expresión oral & $\begin{array}{l}\text { Expresión } \\
\text { escrita }\end{array}$ & $\begin{array}{c}\text { Comprensión } \\
\text { auditiva }\end{array}$ & $\begin{array}{c}\text { Comprensión } \\
\text { lectora }\end{array}$ \\
\hline Chat & & $89 \%$ & & $81 \%$ \\
\hline Grupos de noticias & & $52 \%$ & & $61 \%$ \\
\hline Mensajería & & $84 \%$ & & $59 \%$ \\
\hline Videoconferencia & $91 \%$ & & $92 \%$ & \\
\hline Videosimulaciones & $96 \%$ & $78 \%$ & $87 \%$ & $45 \%$ \\
\hline $\begin{array}{l}\text { Interactividad con redes } \\
\text { sociales }\end{array}$ & & $92 \%$ & & $78 \%$ \\
\hline Listas de distribución & & $45 \%$ & & $53 \%$ \\
\hline $\begin{array}{l}\text { Mapas conceptuales } \\
\text { interactivos }\end{array}$ & & $40 \%$ & & \\
\hline Portafolio del estudiante & & $41 \%$ & & \\
\hline Pizarra compartida & & $42 \%$ & & \\
\hline $\begin{array}{l}\text { Grupos de trabajo } \\
\text { (Talleres) }\end{array}$ & $78 \%$ & $67 \%$ & $82 \%$ & $56 \%$ \\
\hline Wiki & & $59 \%$ & & $61 \%$ \\
\hline \multicolumn{5}{|l|}{ Sindicación RSS } \\
\hline Podcast & $94 \%$ & & $97 \%$ & \\
\hline Blogs & & $67 \%$ & & $69 \%$ \\
\hline Foros & & $87 \%$ & & $65 \%$ \\
\hline
\end{tabular}

Tabla 1. Herramientas 2.0 más funcionales en EVA para la enseñanza de destrezas en segundas lenguas.

\begin{tabular}{|c|c|c|c|c|}
\hline \multirow{2}{*}{$\begin{array}{c}\text { HERRAMIENTAS } \\
2.0\end{array}$} & \multicolumn{4}{|c|}{ DESTREZAS LINGÜÍSTICAS } \\
\hline & Expresión oral & $\begin{array}{l}\text { Expresión } \\
\text { escrita }\end{array}$ & $\begin{array}{l}\text { Comprensión } \\
\text { auditiva }\end{array}$ & $\begin{array}{c}\text { Comprensión } \\
\text { lectora }\end{array}$ \\
\hline Chat & & $49 \%$ & & $41 \%$ \\
\hline \multicolumn{5}{|l|}{ Grupos de noticias } \\
\hline \multicolumn{5}{|l|}{ Mensajería } \\
\hline Videoconferencia & $83 \%$ & & $72 \%$ & \\
\hline Videosimulaciones & $89 \%$ & & $76 \%$ & \\
\hline \multicolumn{5}{|l|}{$\begin{array}{l}\text { Interactividad con redes } \\
\text { sociales }\end{array}$} \\
\hline \multicolumn{5}{|l|}{ Listas de distribución } \\
\hline $\begin{array}{l}\text { Mapas conceptuales } \\
\text { interactivos }\end{array}$ & & $42 \%$ & & \\
\hline \multicolumn{5}{|l|}{ Portafolio del estudiante } \\
\hline $\begin{array}{l}\text { Grupos de trabajo } \\
\text { (Talleres) }\end{array}$ & $79 \%$ & $61 \%$ & $72 \%$ & $45 \%$ \\
\hline Wiki & & $51 \%$ & & \\
\hline \multicolumn{5}{|l|}{ Sindicación RSS } \\
\hline Podcast & $83 \%$ & & $79 \%$ & \\
\hline Blogs & & $57 \%$ & & $42 \%$ \\
\hline Foros & & $82 \%$ & & $44 \%$ \\
\hline
\end{tabular}

Tabla 2. Herramientas 2.0 más funcionales en EVA para la evaluación de destrezas en segundas lenguas.

Píxel-Bit. Revista de Medios y Educación. $N^{o}$ 44. Enero 2014. ISSN: 1133-8482. E-ISSN: 2171-7966. doi: http://dx.doi.org/10.12795/pixelbit.2014.i44.13 
utilidad de las herramientas digitales en el desarrollo de procesos de evaluación de segundas lenguas basados en tareas 2.0 en EVA (ver Tabla 2) (sólo se han reseñado frecuencias iguales o superiores al $40 \%$ de las intervenciones):

Los resultados muestran un sensible descenso del número de herramientas 2.0 con funcionalidad en los procesos de evaluación de destrezas lingüísticas en EVA con respecto a las reseñadas anteriormente en los procesos didácticos.

\section{Discusión.}

Los procesos didácticos y metodológicos que implican la consecución de tareas 2.0 para el desarrollo de destrezas lingüísticas suponen que la tecnología, al igual que la lengua, es un medio para conseguir el producto final, comunicativo y, en este caso, lingüístico-digital. Para ello, hay que escoger las herramientas y recursos 2.0 que mejor se adapten al objetivo final que queremos conseguir. Crear, generar y construir productos comunicativos para mejorar destrezas lingüísticas en segundas lenguas en EVA con posterior proyección en la web implica un desarrollo didáctico y evaluativo basado en la virtualidad de las tareas 2.0; lo que significa dejar de utilizar la web exclusivamente como fuente de input (lo que sin duda también es), y centrarse en las posibilidades que ofrece para crear y publicar contenidos que se integren en un entorno virtual.

Los resultados obtenidos a través del análisis cualitativo de frecuencias en los foros de discusión en línea se agrupan en dos ámbitos asociados a los dos objetivos de la investigación: funcionalidad de herramientas 2.0 en la didáctica y evaluación de segundas lenguas en EVA. Los resultados muestran cómo el alumnado considera mayoritariamente que la didáctica basada en herramientas 2.0 en EVA es participativa, comunicativa, creativa, útil y funcional para el desarrollo de destrezas lingüísticas en segundas lenguas. Con respecto a su utilidad en los procesos evaluadores, consideran mayoritariamente que estos entornos virtuales fomentan la coevaluación, autoevaluación, evaluación continua, evaluación por competencias y evaluación sumativa. Las cinco unidades textuales con mayor frecuencia y representatividad en cada uno de los ámbitos analizados, posicionan la didáctica y la evaluación de segundas lenguas en un nuevo contexto socio-tecnológico y cibercomunicativo que debe generar nuevas formas de enseñar y evaluar en estos entornos digitales.

Los resultados analizados de forma cuantitativa mediante cuestionario muestran que con respecto a la didáctica de destrezas lingüísticas en segundas lenguas, podemos establecer tres categorías de herramientas 2.0: altamente funcionales para todas las destrezas lingüísticas, parcialmente funcionales para un tipo de destrezas y poco o nada funcionales para todas las destrezas. En el primer grupo de herramientas 2.0 altamente funcionales para todas las destrezas reseñamos: videosimulaciones y grupos de trabajo (Talleres). Estas herramientas han sido altamente valoradas con porcentajes superiores al $90 \%$. Es necesario que la didáctica y evaluación de segundas lenguas en EVA integre herramientas y recursos 2.0 que puedan ser útiles y funcionales para la didáctica y la evaluación de forma conjunta. De esa manera, se proyecta una enseñanza fundamentada en 
competencias lingüísticas y digitales que es evaluada con los mismas herramientas 2.0. Las videosimulaciones y los grupos de trabajo en formato talleres son recursos 2.0 que implican el desarrollo funcional de las cuatro destrezas; lo que debería suscitar un mayor empleo de estas herramientas en los procesos didácticos y evaluativos. En un segundo lugar, se reseñan herramientas 2.0 funcionales para un grupo de destrezas específico: expresión oral y comprensión auditiva, donde destacan herramientas 2.0 como la videoconferencia y la creación y audición de podcast. Con respecto a la expresión escrita y comprensión lectora, destacan la interactividad con redes sociales, los foros y los chats. Asimismo, se muestran resultados pocos favorecedores de herramientas 2.0 en tres o cuatro de las destrezas lingüísticas: mapas conceptuales interactivos, portafolio del estudiante, pizarra compartida, wiki y sindicación RSS.

Con respecto a la evaluación de destrezas con herramientas 2.0 en EVA se han apuntado menos herramientas que las reseñadas para la didáctica. La evaluación en formato presencial también es menos rica en propuestas para evaluar que para enseñar. Las herramientas 2.0 con mayor funcionalidad para evaluar cualquiera de las cuatro destrezas es el recurso del trabajo colaborativo en pequeños talleres por ser altamente interactivo y grupal, lo que permite trabajar las cuatro destrezas de forma conjunta. Para la evaluación de la expresión escrita y comprensión lectora, los chats, los blogs y los foros son las herramientas 2.0 mejor valoradas, y para la evaluación de la expresión oral y comprensión auditiva, la videoconferencia, los videosimuladores y la creación y audición de podcast.
Este análisis de la funcionalidad de herramientas 2.0 en la didáctica y evaluación de segundas lenguas constata la importancia de diseñar los EVA dependiendo del tipo de enseñanza al que están destinados. El diseño de los EVA debe fomentar en el alumnado también el desarrollo de una cierta alfabetización en el uso de estas herramientas, que necesitará a su vez para elaborar tareas comunicativas digitales, las tareas 2.0. Basándonos en los resultados obtenidos en la investigación creemos que se debe reorientar el papel docente y discente en los EVA. Las funciones didácticas de los profesores en EVA para la enseñanza de segundas lenguas en el espacio Europeo de Educación Superior deben caracterizarse por:

a) Crear un entorno adecuado al aprendizaje mediante la creación de una atmosfera creativa, solidaria, participativa y altamente colaborativa.

b) Realizar el control y moderación de las tareas 2.0 e iniciar procesos de automoderación entre los estudiantes.

c) Proporcionar las condiciones en las que los alumnos entiendan la funcionalidad y su papel creativo en el desarrollo de las tareas 2.0 para el aprendizaje de destrezas lingüísticas.

d) Poner de relieve y explicitar lo que los alumnos tienen que hacer y cómo, así como distribuir las distintas asignaciones de control, mantenimiento y creación de contenidos 2.0 basados en destrezas lingüísticas prioritarias (leer, escuchar, hablar...).

e) Juzgar las aportaciones de los alumnos y, cuando corresponda, realizar la evaluación de las mismas.

f) Realizar las demostraciones necesarias del producto a desarrollar y proveer modelos lingüísticos que faciliten el aprendizaje y el desarrollo de las tareas 2.0. 
Desde el punto de vista discente, la creación de tareas 2.0 permite adquirir una conciencia y control de aprendizaje progresivamente a través de las siguientes facetas desarrolladas en cooperación con el profesor:

a) Sentido de la competencia. Los alumnos van adquiriendo conciencia de su propio aprendizaje al tener que desarrollar contenidos en los que su propia creación es fundamental. En este sentido, la interacción entre su producción y el control del profesor (a través de correcciones antes de la edición y publicación del output) son fundamentales en el aprendizaje.

b) Control del comportamiento lingüístico. El alumno usa las estructuras de manera repetida hasta adquirir una competencia en las mismas.

c) Consciencia del cambio. El alumno puede modificar sus propios objetivos, por lo que tiene que justificar su evolución y adquiere la conciencia de la importancia y la justificación del cambio.

d) Sentimiento positivo sobre los propios logros. Esta sensación evidentemente motivadora es muy importante tanto en el aprendizaje general como en el de segundas lenguas.

Los tipos de pruebas mediante tareas 2.0 requieren que los estudiantes se involucren en algún tipo de conducta que simule, de la forma más fiel posible, un uso de la lengua meta orientado a un objetivo o situación. En esta investigación, se han mostrado como altamente productivos recursos 2.0 en metodología y evaluación que requieren de actuaciones de acuerdo con elementos de criterio predeterminado y relacionado con el mundo real (procesos y productos de la tarea), y a niveles de criterio (estándares auténticos relacionados con el éxito de la tarea, así como su recursividad en el espacio web). Por lo tanto, y siendo consecuentes con estas metodologías basadas en las tareas 2.0, la evaluación de las mismas debe seguir unos parámetros similares a su didáctica, lo que significa que este proceso evaluativo, de marcado carácter pedagógico, ha de construirse a partir de los siguientes principios:

a) Uso individualizado y contextualizado de las herramientas evaluativas con el foco en las necesidades del estudiante con respecto a las destrezas lingüísticas.

b) Coevaluación que fomente una evaluación horizontal e incluya al aprendiente en los procesos de monitorización y retroalimentación de su aprendizaje.

c) Evaluación procesual para evaluar no sólo el producto final comunicativo de las tareas 2.0, sino también todo el proceso de consecución de este (vídeo y podcast, por ejemplo).

d) (Co)Responsabilidad en la evaluación para compartir entre los miembros de la comunidad de aprendizaje, lo que la convierte en un proceso más rico y variado.

\section{Imitaciones de la investigación y prospectiva.}

Los resultados obtenidos se contextualizan en las enseñanzas de un Máster de la UNED y se limitan al análisis de los recursos 2.0 en entornos virtuales para la plataforma aLF operativa en la UNED. Tanto la muestra de sujetos analizados como los estudios en los que se han aplicado los instrumentos de recogida de datos, se podrían ampliar en un futuro longitudinalmente para ampliar los resultados y derivar nuevas aproximaciones y discusiones. Los resultados obtenidos se deberían confrontar en el futuro con otras 
investigaciones en otras Universidades que basan sus enseñanzas en otras plataformas interactivas para ir proponiendo nuevas vías de actuación en la didáctica y evaluación 2.0 de segundas lenguas en EVA.

\section{Conclusiones.}

El trabajo con modelos lingüísticos nos señala la importancia que, comparada con la información, tiene la comunicación en los procesos de enseñanza-aprendizaje de segundas lenguas. No obstante, en esta búsqueda hemos de ser prudentes y superar el entusiasmo primerizo si se trata de aplicar las TIC a la adquisición de segundas lenguas. La adquisición de segundas lenguas en EVA tiene que implicar, además de la adquisición de destrezas asociadas a la competencia linguiística, la adquisición de competencias telemáticas que promueven principalmente la participación activa de los estudiantes en su proceso de aprendizaje, en el andamiaje del conocimiento, en el desarrollo del pensamiento creativo y crítico, en el proceso de comunicación intercultural y en el aprendizaje flexible que les permite entender temas sociales y lingüísticos relacionados con la tecnología. Asimismo, la trayectoria evaluadora en segundas lenguas en EVA debe ir enfocada a un tipo de evaluación más autónoma. Los proyectos de trabajo basados en tareas 2.0 de realización individual y/o de grupo deben incluir un trabajo de análisis, reflexión, autoevaluación y/o coevaluación de los participantes en el proyecto para seguir aprendiendo la lengua fuera del contexto formal de aprendizaje y a lo largo de la vida. A modo de resumen, podemos concluir diciendo que, en base a los resultados de la investigación, las tareas 2.0 mediante herramientas digitales (vídeo, podcast, chats, intercambio de archivos, etc.) y su evaluación es un excelente motor para el desarrollo de los aspectos más importantes del aprendizaje y la evaluación de una segunda lengua; no limitándose el formato en EVA a ser únicamente un contexto para el aprendizaje, sino auténticos motores que integran tareas, funciones y colaboración entre los distintos agentes del aprendizaje (alumnos y profesores), además de ser un medio muy motivador para reforzar otras áreas educativas y mejorar el conocimiento general del mundo que tienen los estudiantes.

\section{Referencias bibliográficas.}

Arandia, M., Alonso-Olea, M.J. \& MartínezDomínguez, I. (2010). La metodología dialógica en las aulas universitarias. Revista de Educación, 352, 309-329.

Area, M. \& Adell, J. (2009). E-learning: enseñar y aprender en espacios virtuales. En J. de Pablos Pons (Coord.). Tecnología educativa. La formación del profesorado en la era de Internet. (pp. 391-424). Málaga: Ediciones Aljibe.

Arnold, N. (2007). Reducing foreign language communication apprehension with computer-mediated communication: A preliminary study. System, 35, 469-486. http:/ /dx.doi.org/10.1016/j.system.2007.07.002

Bachman, L.F. (2000). Modern language testing at the turn of the century: assuring that what count counts. Language Testing, 17(1), 1-42.

Bolívar, A., Domingo, J. \& Fernández, M. (2001). La investigación biográficonarrativa en educación. Enfoque y metodología. Madrid: La Muralla.

Boneu, J.M. (2007). Plataformas abiertas de e-learning para el soporte de contenidos educativos abiertos. Revista de Universidad 
y Sociedad del Conocimiento (RUSC), 4(1), 36-47.

Bugler, D. \& Hunt, A. (2002). Implementing Task-base Language Teaching. En J. Richards \& W. Renandya (Eds.). Methodology in Language Teaching: An Anthology of Current Practice. (pp. 205-213). Cambridge: Cambridge University Press.

Cabero, J. (Dir.) (2010). Usos del e-learning en las Universidades Andaluzas: Estado de la cuestión y análisis de buenas prácticas. Sevilla: GID.

Campbell, N. (2007). Bringing ESL students out of their shells: Enhancing participation through online discussion. Business Communication Quarterly, 70(1), 37-43. doi: 10.1177/108056990707000105

Chase, S.E. (1995). Taking narrative seriously: Consequences for method and theory in interview studies. The Narrative Studies of Lives, 3(1), 1-26.

Clandinin, D.J. \& Connelly, F.M. (1994). Personal experience methods. En N.K. Denzin \& Y.S. Lincoln (Eds.). Handbook of qualitative research.(pp. 413-427). California: Sage.

Coniam, D. (2008). Evaluating the language resources of chatbots for their potential in English as a second language. $\operatorname{Re} C A L L, 20(1)$, 98-116. doi: 10.1017/S0958344008000815

Copley, J. (2007). Audio and video podcasts of lectures for campus-based students: production and evaluation of student use. Innovations in Education and Teaching International, 44, 387-399. doi:10.1080/ 14703290701602805

De Benito, B. \& Salinas, J. (2008). Los entornos tecnológicos en la Universidad. Píxel-Bit. Revista de Medios y Educación, 32, 83-100. Recuperado de: http:// www.sav.us.es/pixelbit/pixelbit/ articulos/ n32/6.pdf
De Freitas, S. \& Neumann, T. (2009). The use of exploratory learning for supporting immersive learning in virtual environments. Computers \& Education, 52, 343-353. doi: 10.1016/j.compedu.2008.09.010

De Pablos, J., Colas, P. \& Villaciervos, T. (2010). Políticas educativas, buenas prácticas y TIC en la comunidad autónoma andaluza. Teoría de la Educación. Educación y Cultura en la Sociedad de la Información, 11(1), 180-202.

Del Moral, M.E. \& Villalustre, L. (2012). Didáctica universitaria en la era 2.0: competencias docentes en campus virtuales. Revista de Universidad y Sociedad del Conocimiento (RUSC), 9(1), 36-50. Recuperado de http://rusc.uoc.edu/ojs/ index.php/rusc/article/view/v9n1-moralvillalustre/v9n1-moral-villalustre

Ellis, R. (2009). Task-Based Language Teaching: Sorting out the Misunderstandings International. Journal of Applied Linguistics, 19, 221-246. doi: 10.1111/j.1473-4192.2009.0023

Fallahkhair, S., Pemberton, L. \& Griffiths, R. (2007). Development of a cross-platform ubiquitous language learning service via mobile phone and interactive television. Journal of Computer Assisted Learning, 23(4), 312-325. doi: 10.1111/j.13652729.2007.00236.x

Harrison, R. \& Thomas, M. (2009). Identity in online communities: Social networking sites and language learning. International Journal of Emerging Technologies \& Society, 7(2), 109-124.

Hernández, R., Fernández, C. \& Baptista, P. (2006). Metodología de la investigación. México: McGraw-Hill.

Hernández, F. \& Vergara, O. (2004). ¿Son útiles los diarios de la clase? Experiencia con el componente de investigación en el aula en 
la Universidad del Valle. Lenguaje, 32, 212232.

Hislope, K. (2008). Language learning in a virtual world. The International Journal of Learning, 15(11), 51-58.

Jonassen, D.H. (1995). Supporting communities of learners with technology: A vision for integrating technology in learning in schools. Educational Technology, 35(4), 60-62.

Lan, Y.J., Sung, Y.T. \& Chang, K.E. (2007). A mobile-device-supported peer-assisted learning system for collaborative early EFL reading. Language Learning \& Technology, 11(3), 130-151.

Martínez-Miguélez, M. (2006). Validez y confiabilidad en la metodología cualitativa. Paradigma, 2, 7-34.

Nevgi, A. \& Tirri, K. (2003). In search of good virtual teaching. The advantages and disadvantages to learning in virtual environments. Students' experiences and teachers' evaluations. Research in Educational Sciences, 15, 9-17.

Ortega, L. (september, 2009). Tasks and technology in language learning: Elective affinities and (dis)encounters. Plenary delivered at the 3rd International Task-Based Language Teaching Conference. Lancaster.

Reeder, K. (2010). Edubba: Real-world writing tasks in a virtual world. En M. Thomas \& H. Reinders (Eds.). Task-based language learning and teaching with technology. (pp. 176-196). London \& New York: Continuum.

Sharples, M. (Ed.) (2006). Big issues in mobile learning. Report of a workshop by the Kaleidoscope Network of Excellence Mobile Learning Initiative. United Kingdom: University of Nottingham.

Schön, D. A. (1992). La formación de profesionales reflexivos. Hacia un nuevo diseño de la enseñanza y el aprendizaje en las profesiones. Barcelona: Paidós/M.E.C.

Solano, I. \& Sánchez, M.M. (2010). Aprendiendo en cualquier lugar: el podcast educativo. Píxel-Bit. Revista de Medios y Educación, 36, 125-139. Recuperado de http:/ /www.sav.us.es/ pixelbit/pixelbit/articulos/ n36/10.pdf

Thomas, M. \& Reinders, H. (Eds.) (2010). Task-based language learning and teaching with technology. London \& New York: Continuum.

Valle-López, J.M. (2010). El Proceso de Bolonia: un nuevo marco de aprendizaje para la educación superior. En J. Paredes \& A. de la Herrán (Coords.). Cómo enseñar en el aula universitaria. (pp. 47-58). Madrid: Pirámide.

Vázquez-Cano, E. \& Sevillano, M. L. (2011). Educadores en Red. Elaboración y edición de materiales audiovisuales para la enseñanza. Madrid: UNED-Ediciones Académicas.

Willis, J. \& Willis, D. (2009). Task-based language teaching: Some questions and answers. The Language Teacher, 33(8), 3-8.

Fecha de recepción: $\quad$ 08-03-2012

Fecha de evaluación: 25-05-2012

Fecha de aceptación: 14-07-2012 